\section{Predicting difficult laryngoscopy}

\section{To the Editor:}

Rose and Cohen' report the grading of the view at laryngoscopy in 3325 patients by 25 certified anaesthetists. No details are given in regard to the conduct of laryngoscopy. No mention is made of a protocol, or control measures, to ensure that every participant (i) performed the best possible laryngoscopy, (ii) obtained the best possible laryngeal view, (iii) obtained the best possible laryngoscopy grading, in every patient. These cannot be just left to chance nor assumed. ${ }^{2}$ The perfect performance of laryngoscopy and grading by all participants on all patients is central to the success or failure of any such research work. Retrospective discussion is no substitute for prospective design and reliability.

In order to assess the results the reader needs to know, in regard to Methods, about "sniffing" position, good relaxation, firm forward laryngoscope traction, use of Macintosh 4 blade if Macintosh 3 blade is inadequate, placement of laryngoscope blade posterior to the epiglottis (if placement anterior is unsuccessful), firm backward thyroid cartilage pressure, trial of increased neck flexion, etc. Also it must be clear that misinterpretations and inaccuracies of grading, particularly Grade III, have been avoided. ${ }^{3,4}$ For example the long wide floppy epiglottis is invariably easily converted to Grade I using one or more of Macintosh 4 blade, laryngoscope blade posterior to the epiglottis, or backward thyroid cartilage pressure. Alternatively, in patients with a narrow jaw and lesser mouth opening all of these measures and increased neck flexion may be needed to convert to a Grade II view. It is only when just the tip of the epiglottis is visible that all fail to improve the view and grading.

At laryngoscopy what one sees depends on what one does. It is as essential to define input i.e., the performance of laryngoscopy, as it is outcome because the former influences the latter. Rose and Cohen's ${ }^{1}$ revised anaesthetic record does not really cover this aspect which must continue to affect the results.

R.Williamson MB FFARCS

Durban, South Africa

\section{REFERENCES}

1 Rose $D K$, Cohen $M M$. The incidence of airway problems depends on the definition used. Can J Anaesth 1996; 43: $30-4$.

2 Williams KN, Carli F, Cormack RS. Unexpected, difficult laryngoscopy: a prospective survey in routine general surgery. Br J Anaesth 1991; 66: 38-44.

3 Williamson $R$. Grade III laryngoscopy - which is it? (Letter) Anaesthesia 1988; 43: 424.

4 Williamson $R$. Difficult laryngoscopy (Letter). $\mathrm{Br} \mathrm{J}$ Anaesth 1992; 68:117-8

\section{REPLY}

This reader seeks more information on the conduct of laryngoscopy. He stresses the importance of documentation of "the perfect performance of laryngoscopy and grading by all participants. "No one can argue with his suggestions that patient position, degree of relaxation, blade type, size, placement, and traction, and laryngeal pressure influence the "best possible view." We agree that if routine anaesthetic practice encom- passed these suggestions, laryngoscopy view would be optimal.

However, our study described the reality of clinical practice. The description of the methodology to ensure "best possible view" was not specified. We assumed that all anaesthetists would optimize laryngeal view prior to tracheal intubation. This is an integral part of all our training programmes. However, we clearly stated in the discussion the limitations of this assumption. Factors which prevented optimal laryngeal view would lead to an overestimate of the incidence of the Grade 3 and 4 laryngoscopy view (from the modified diagrams of Cormack and Lehane). The main purpose of our study was to determine if the view at laryngoscopy defined by four grades on the modified diagrams and the number of laryngoscopy attempts measured different dimensions of airway difficulty. Using the methodology in our study we concluded that they did. There was wide variation in defining the extent of the airway problem. These findings led us to revise our documentation of airway management. We now include information on the blade size and type, use of stylet, and laryngeal pressure (incorrectly noted as cricoid pressure in Figure 4 of the original article). We have expanded the grading system from four categories to five, replaced the pictures with descriptive phrases, and indicated that anaesthetists should record the best view at laryngoscopy.

For a similar patient population (May 1, 1995 to April 30, 1996), there were an additional 6,477 patients who had direct laryngoscopy with attempted tracheal intubation following general anaesthesia. The rate of view of epiglottis or no view of epiglottis was $1.5 \%, 23$ laryngoscopies $10.7 \%$, and failure to intubate $0.3 \%$. This rate of poor laryngoscopy view defined by our new criteria as Grade $D$ or $E$ was decreased from our previous study (10.1\%) but identical to the frequency reported in a prospective study by Wilson.' Like our previous study, only a relatively small percentage $(26.3 \%)$ with view of only epiglottis or no view of epiglottis had $>3$ laryngoscopies (previous study $15.8 \%$ ). However, for those patients with 23 laryngoscopies, only $22.1 \%$ of those in the recent study compared with $84.1 \%$ previously had a view of only epiglottis or no epiglottis. The notation of best view on the record and the new grading system may have contributed to these reduced frequencies.

With this new information our conclusion remains the same. View at laryngoscopy and number of laryngoscopy attempts measure different dimensions of airway difficulty. We also think that future modifications of the recording of ainway management should include additional criteria, e.g., time required for tracheal intubation. Objective information will continue to add to our knowledge of the "extent of the problem."

D.K. Rose MD FRCPC

M.M Cohen MD FRCPC

Toronto, Ontario M5B 1W8

REFERENCE

1 Wilson ME, Spiegelhalter D, Robertson JA, Lesser $P$ Predicting difficult intubation. Br J Anaesth 1988; 61: $211-6$. 